\title{
ESTUDO DA MORBIMORTALIDADE CIRÚRGICA, DA SOBREVIDA E DOS FATORES PROGNÓSTICOS DOS PACIENTES PORTADORES DE SARCOMAS PRIMÁRIOS DO RETROPERITÔNIO
}

\section{SURGICAL MORBIMORTALITY, SURVIVAL AND PROGNOSTIC FACTORS OF PATIENTS WITH PRIMARY RETROPERITONEAL SARCOMAS}

\author{
Carlos Eduardo R. Santos,TCBC-RJ'1 ; Orlando Marques Vieira,TCBC-RJ'2; \\ Mauro Monteiro Correa, TCBC-RJ ${ }^{3}$; Gustavo Santos Stoduto de Carvalho ${ }^{4}$; \\ Felipe Manzani' $;$ Jurandir de Almeida Dias ${ }^{6}$
}

\begin{abstract}
RESUMO: Objetivo: Avaliar a morbimortalidade, sobrevida e os fatores prognósticos dos sarcomas primários do retroperitônio. Método: Análise retrospectiva de 59 pacientes com sarcoma de retroperitônio, operados na Seção de Cirurgia Abdomino-Pélvica do Instituto Nacional de Câncer no período de junho de 1992 a julho de 2003. Resultados: As queixas mais comuns foram dor abdominal e massa abdominal. A taxa de ressecabilidade foi de $74,57 \%$ e a de radicalidade entre os ressecados de $48,88 \%$. Houve dois óbitos pós-operatórios $(3,38 \%)$ e 12 complicações pós-operatórias (20,33\%). Os leiomiossarcomas e os lipossarcomas foram os mais incidentes. O grau de diferenciação tumoral mais freqüente foi o G3 $(38,98 \%)$ e o diâmetro tumoral médio, de $20,4 \mathrm{~cm}$. A sobrevida global foi de $49 \%$ em dois anos e $20 \%$ em cinco anos, e a mediana de sobrevida livre de doença foi de 23 meses. À análise univariada, o diâmetro do tumor (> ou <= $12 \mathrm{~cm}$ ), o grau de diferenciação tumoral ([G1 + G2] X [G3 + G4]), a ressecção radical (R0) ou paliativa $(\mathrm{R} 1+\mathrm{R} 2)$, a hemotranfusão no ato operatório e a re-ressecção, mesmo que paliativa, nos casos de recidiva ou persistência de doença $(\mathrm{n}=52)$, foram significativos para sobrevida $(\mathrm{p}=0,0267,0,048,0,0001,0,022$ e 0,0003 , respectivamente). Conclusão: No momento, somente o diagnóstico precoce, a cirurgia radical R0, a ausência de hemotransfusão intra-operatória e a re-ressecção nos casos de recidiva ou persistência de doença possibilitarão a sobrevida a longo prazo (Rev. Col. Bras. Cir. 2005; 32(5): 251-255).
\end{abstract}

Descritores: Sarcoma; Neoplasias retroperitoneais/cirurgia; Indicadores de morbidade e mortalidade; Taxa de sobrevida; Prognóstico.

\section{INTRODUÇÃO}

Sarcomas de partes moles são tumores raros, que representam $1-2 \%$ de todos os tumores malignos sólidos. Somente $10-20 \%$ desses tumores estão localizados no espaço retroperitoneal, e sua incidência é de 0,3-0,4 casos por 100.000 habitantes, nos Estados Unidos ${ }^{1}$. Não há dados específicos sobre os sarcomas primários do retroperitônio no registro brasileiro de tumores, e nos Estados Unidos 1.000 casos novos de sarcoma de retroperitônio são diagnosticados anualmente. Em grandes séries de pacientes com neoplasia de retroperitônio, mais de 50\% mostraram ser de origem mesenquimal ${ }^{2}$. A biologia desses tumores é semelhante à de outros sarcomas e com grande diferença para os cânceres do trato gastrointestinal. Metástases para linfonodos são muito raras, e somente Zorig et al. ${ }^{3}$ descreveram esse achado em $20 \%$ dos casos. Metástases à distância (pulmão e fígado) são também infreqüentes e ocorrem mais em tumores de alto grau, após longo tempo de crescimento do tumor primário ${ }^{4}$.
Devido a seu comportamento biológico e a sua localização anatômica, estes tumores tornam-se sintomáticos tardiamente e com freqüente invasão de estruturas retroperitoneais contíguas. Esses fatores podem tornar a ressecção cirúrgica difícil ou impossível.

Até o momento, o tratamento quimioterápico dos sarcomas de retroperitônio não tem sido eficaz, e a radioterapia é limitada pela toxicidade de estruturas adjacentes intra-abdominais ${ }^{5}$. A ressecção completa é a terapêutica mais eficaz para tumores primários selecionados e doença recidivante. $\mathrm{O}$ prognóstico dos pacientes com sarcomas de retroperitônio é ruim, com taxa de recidiva local alta e taxa de sobrevida baixa em cinco anos. Em contraste com sarcoma de partes moles de outras localizações, somente a cirurgia tem influência significativa na sobrevida de pacientes com sarcomas do retroperitônio.

Este estudo visa a determinar a morbimortalidade, a sobrevida e a identificação de possíveis fatores prognósticos dos sarcomas primários do retroperitônio operados no Instituto Nacional de Câncer, por uma análise uni e multivariada dos resultados.

1. Cirurgião Oncológico do INCA; Professor Assistente de Cirurgia da UNIGRANRIO.

2. Professor Emérito do Departamento de Cirurgia da Faculdade de Medicina da UFRJ.

3. Cirurgião Oncológico do INCA; Chefe da Cadeira de Cirurgia do Adulto e do Idoso da UNIGRANRIO.

4. Residente de Cirurgia Oncológica do INCA.

5. Acadêmico de Medicina da UNIGRANRIO.

6. Chefe do Serviço de Cirurgia abdômino-pélvica do INCA.

Recebido em: 16/03/2005

Aceito para publicação em: 26/09/2005

Conflito de interesse: nenhum

Fonte de financiamento: nenhuma

Trabalho realizado na Seção de cirurgia abdômino-pélvica do Instituto Nacional de Câncer (INCA). Tese apresentada ao término do curso de mestrado em cirurgia abdominal do programa de pós-graduação em Cirurgia Geral do Departamento de Cirurgia da UFRJ. 


\section{MÉTODO}

Analisamos retrospectivamente 59 prontuários de pacientes portadores de sarcoma primário do retroperitônio, operados no Instituto Nacional de Câncer (INCA), identificados na base de dados informatizada do serviço de anatomia patológica, e operados de junho de 1992 a julho de 2003.

Os critérios de inclusão foram o diagnóstico histológico de sarcoma, localização primária no retroperitônio e tratamento cirúrgico. Como, por definição, tumor de retroperitônio é aquele que provem dos tecidos que preenchem o espaço retroperitonial, foram excluidos os tumores de rim, ureter e supra-renal.

Foram avaliados os tipos de procedimentos realizados e sua morbimortalidade cirúrgica. A sobrevida global foi calculada através do método de Kaplan Meier. Para a comparação univariada dos tempos de sobrevida global, considerando todas as variáveis em estudo, foi aplicado o teste de Log Rank, adotando-se o nível de significância de 5\% de probabilidade $(p<0,05)$.

A análise multivariada foi realizada através do método de regressão logística do teste de Wald das variáveis significativas encontradas na análise univariada.

Quanto ao grau histológico, foram considerados G1 aqueles tumores bem diferenciados, G2 os moderadamente diferenciados, $\mathrm{G} 3$ os pouco diferenciados, $\mathrm{G} 4$ os indiferenciados e GX aqueles em que não houve determinação.

Quanto à radicalidade da cirurgia, foi considerada R0 a cirurgia radical sem evidência de doença residual, R1 a cirurgia paliativa com evidência de doença residual microscópica e R2 a cirurgia paliativa com evidência de doença macroscópica. Os pacientes com cirurgias R1 e R2 foram considerados com persistência de doença.

\section{RESULTADOS}

Dos 59 pacientes operados no período, 23 eram homens e 36 mulheres; 38 brancos, seis negros e 15 pardos, com idade média de 46 anos e mediana de 50 anos (seis a 72 anos). História familiar de câncer esteve presente em 21 casos $(35,59 \%)$. As queixas mais comuns foram dor abdominal (31)e massa abdominal (17). TC foi realizada em 53, USG em 24 e RM em seis no pré-operatório.

A taxa de ressecabilidade foi $74,57 \%$ ( 44 pacientes) e a de radicalidade entre os ressecados de $48,88 \%$ (22 pacientes). Vinte e nove $(64,44 \%)$ pacientes tiveram ressecções de órgãos associados, totalizando 70 órgãos ressecados em associação, e dos quais apenas $22(37,14 \%)$ estavam realmente invadidos na análise histopatológica. O tempo médio de cirurgia foi de quatro horas e oito minutos. Em 35,59\% (21 pacientes) houve hemotransfusão (500-3000 ml / média de 1.166,66 ml), e nesses casos o tempo médio da cirurgia foi de cinco horas e 33 minutos. Houve dois óbitos pós-operatórios (3,38\%), ambos em ressecções paliativas, sendo o primeiro caso devido à embolia pulmonar no terceiro dia pós-operatório e o segundo caso devido a complicações técnicas por lesão de aorta, com falecimento no pós-operatório imediato. Houve 12 complicações (20,33\%) (quatro sangramentos, uma laceração duodenal, uma obstru-

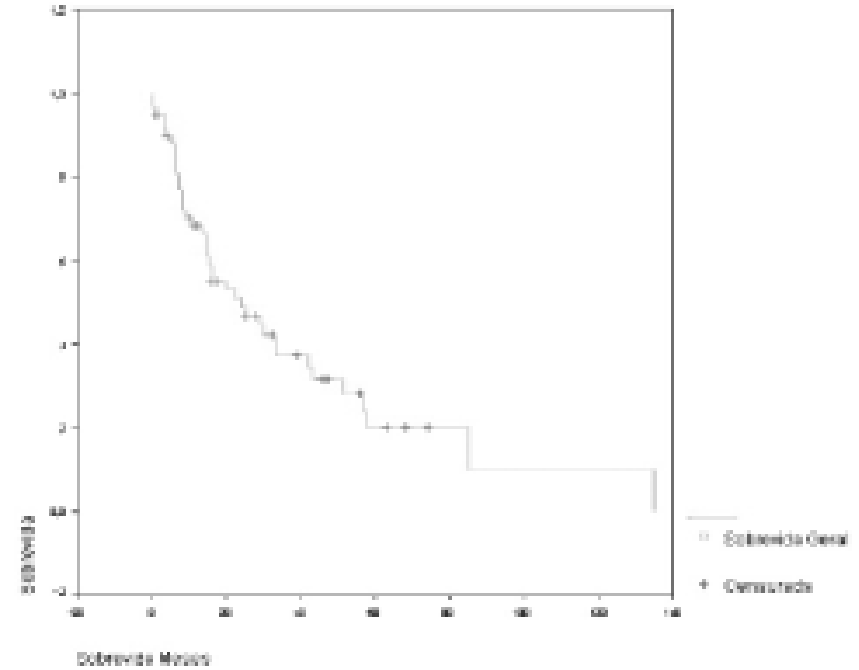

Gráfico 1 - Analise da sobrevida global de pacientes com sarcoma de retroperitônio operados.

ção intestinal, uma evisceração, uma lesão esplênica, duas neutropenias, uma insuficiência respiratória e uma trombose venosa profunda), levando a quatro reoperações associadas (8,9\%). Os leiomiossarcomas (16 paciente) e os lipossarcomas (15 pacientes) foram os mais incidentes, representando juntos $52,54 \%$ do total de pacientes. $\mathrm{O}$ grau de diferenciação tumoral mais freqüente foi o G3 [23 pacientes $(38,98 \%)$ ] e o diâmetro tumoral médio de $20,4 \mathrm{~cm}$ (quatro a $52 \mathrm{~cm}$ ) e mediano de $18 \mathrm{~cm}$. Cinco pacientes $(8,47 \%)$ tiveram tratamento neoadjuvante $(\mathrm{QT}=1, \mathrm{RXT}=1, \mathrm{QT}+\mathrm{RXT}=3)$ e 26 pacientes $(44,06 \%)$ tiveram tratamento adjuvante $(\mathrm{RXT}=13, \mathrm{QT}=9, \mathrm{QT}+\mathrm{RXT}=4)$. Dos pacientes que sofreram ressecções radicais (21 pacientes), 16 recidivaram $(72,72 \%)$, seguindo o padrão hematogênico em sete casos, local em quatro casos, peritoneal em seis casos e linfonodal em dois casos, sendo três pacientes com recidiva em dois padrões distintos e concomitantes. Treze desses pacientes $(81,25 \%)$ foram submetidos à re-ressecções, das quais oito consideradas radicais $\mathrm{R} 0(61,53 \%)$.

A re-ressecção no total de pacientes ressecados (45 pacientes) aconteceu em 21 casos $(46,66 \%)$, sendo única em 16 , dupla em três, tripla em dois e radical em 10 pacientes desse somatório total, não gerando óbito.

A sobrevida global foi de $49 \%$ em dois anos e $20 \%$ em cinco anos, pelo método de Kaplan-Meier (Gráfico 1 e Tabela1).

Tabela 1 - Sobrevida global.

\begin{tabular}{cc}
\hline Tempo (Meses) & Px \\
\hline $0-6$ & 0,80 \\
$6-13$ & 0,66 \\
$13-20$ & 0,53 \\
$20-24$ & 0,49 \\
$24-30$ & 0,42 \\
$30-33$ & 0,37 \\
$33-42$ & 0,34 \\
$42-51$ & 0,28 \\
$51-57$ & 0,24 \\
$57-58$ & 0,20 \\
\hline
\end{tabular}


Tabela 2 - Análise univariada da comparação entre os tempos de sobrevida global e as variáveis abaixo.

\begin{tabular}{|c|c|c|}
\hline Variáveis & $\begin{array}{l}\text { Nível de Significância } \\
\log \operatorname{Rank}(\mathbf{p})\end{array}$ & Significância \\
\hline $\begin{array}{l}\text { História Familiar } \\
\text { (Sim X Não) }\end{array}$ & $P=0,25$ & $\begin{array}{l}\text { N.S. } \\
\text { N.S. }\end{array}$ \\
\hline Sintomas (Massa X Dor) & $P=0,16$ & N.S. \\
\hline Idade $(=<$ Me $\mathrm{X}>\mathrm{Me})$ & $P=0,64$ & N.S. \\
\hline Sexo (Masc. X Fem.) & $P=0,22$ & N.S. \\
\hline Raça (Brancos X Não Brancos) & $P=0,41$ & N.S. \\
\hline $\begin{array}{l}\text { Ressecção } \\
{[\mathrm{R} 0 \mathrm{X}(\mathrm{R} 1+\mathrm{R} 2)]}\end{array}$ & $P=0,0001$ & Significativo \\
\hline $\begin{array}{l}\text { Tratamento Adjuvante } \\
\text { (Não X Qt e/ou Rxt) }\end{array}$ & $P=0,47$ & N.S. \\
\hline $\begin{array}{l}\text { Tipo Histológico } \\
\text { (Leiomossarcoma / Lipossarcoma / Outros) }\end{array}$ & $P=0,97$ & N.S. \\
\hline $\begin{array}{l}\text { Diâmetro do Tumor } \\
(\leq 12 \mathrm{~cm} X>12 \mathrm{~cm})\end{array}$ & $P=0,0267$ & Significativo \\
\hline $\begin{array}{l}\text { Grau de Diferenciação } \\
{[(\mathrm{G} 1+\mathrm{G} 2) \mathrm{X}(\mathrm{G} 3+\mathrm{G} 4)]}\end{array}$ & $P=0,048$ & Significativo \\
\hline $\begin{array}{l}\text { Hemotransfusão } \\
\text { (Sim X Não) }\end{array}$ & $P=0,022$ & Significativo \\
\hline $\begin{array}{l}\text { Órgãos Associados } \\
\text { (Sim X Não) }\end{array}$ & $P=0,64$ & N.S. \\
\hline $\begin{array}{l}\text { Re-ressecção no caso de recidiva ou } \\
\text { persistência da doença (Sim X Não) }\end{array}$ & $P=0,0003$ & Significativo \\
\hline
\end{tabular}

$M e=$ mediana

$Q t=$ quimioterapia

$R r t=$ radioterapia

$N . S .=$ não significativo

A mediana de sobrevida livre de doença nos pacientes que sofreram ressecções radicais (22 pacientes), levando-se em conta o maior período livre de doença nos pacientes que sofreram mais de uma ressecção, foi de 23 meses e a média de 28,18 meses. Foram incluídos no cálculo da sobrevida os óbitos pós-operatórios. Dezoito pacientes encontram-se em acompanhamento ambulatorial, dos quais nove com evidência de doença e nove sem evidência de doença, com tempo de seguimento variando de zero a 135 meses. Sete dos pacientes sem evidência de doença nunca apresentaram recidiva.

$\mathrm{Na}$ análise univariada, não demonstraram significância estatística quanto à sobrevida global: história familiar positiva para câncer, sintomas iniciais de dor ou massa, idade maior ou menor que a mediana (50 anos), sexo, raça branca ou não, realização de tratamento adjuvante (radioterapia e/ou quimioterapia), tipo histológico e ressecção associada de outros órgãos. Já o diâmetro do tumor ( $>$ ou $<=12 \mathrm{~cm}$ ), o grau de diferenciação tumoral [(G1 + G2)] X [(G3 + G4)], a ressecção radical (R0) ou paliativa $(\mathrm{R} 1+\mathrm{R} 2)$, a hemotranfusão no ato operatório, e a reressecção, mesmo que paliativa, nos casos de recidiva ou persistência de doença $(\mathrm{N}=52)$, foram significativos $(p=0,0267$, $0,048,0,0001,0,022$ e 0,0003 , respectivamente) (Tabela 2).

$\mathrm{Na}$ análise multivariada de regressão logística pelo teste de Wald em relação ao diâmetro do tumor, grau de dife- renciação celular, hemotransfusão, radicalidade da ressecção e re-ressecção, somente a re-ressecção mostrou uma tendência para variável independente $\operatorname{com} p=0,0704$ (Tabela 3).

\section{DISCUSSÃO}

$\mathrm{Na}$ análise dos dados obtidos, notamos predominância de mulheres brancas com idade mediana de 50 anos, sem história familiar de câncer e com dor abdominal como principal sintoma. Nenhum desses fatores foi significativo na análise estatística quanto ao prognóstico, em conformidade com a literatura ${ }^{2,6,7}$.

A tomografia computadorizada foi o exame pré-operatório mais freqüentemente realizado, por permitir uma análise detalhada do tumor e sua relação com as estruturas adjacentes, sendo somente solicitada a ressonância magnética nos casos de dúvida em relação ao comprometimento vascular.

As taxas de ressecabilidade $(74,57 \%)$ e de radicalidade entre os ressecados $(48,88 \%)$ foram superiores à média da Seção de Cirurgia Abdomino-Pélvica do Instituto Nacional de Câncer para o conjunto de tumores sólidos do aparelho digestivo ${ }^{8,9}$, assim como a mortalidade foi baixa seguindo essa comparação (3,38\%). É interessante notar que, 
Tabela 3 - Análise multivariada da comparação entre a sobrevida global e as variáveis com significância estatística na análise univariada.

\begin{tabular}{lcccc}
\hline Variável & $\boldsymbol{\beta i}$ & Odds Ratio & Intervalo de Confiança & $\mathbf{p}$ \\
\hline Radicalidade da ressecção & 0,9325 & 1,0860 & 0,$1606 ; 7,3431$ & 0,9325 \\
Maior diâmetro do tumor & 0,4632 & 2,0091 & 0,$3114 ; 12,9610$ & 0,4632 \\
Grau de diferenciação celular & 1,4782 & 4,4098 & 0,$6165 ; 31,5412$ & 0,1394 \\
Hemotranfusão & 0,3234 & 1,4013 & 0,$1814 ; 10,8275$ & 0,7464 \\
Re-ressecção & $\mathbf{0 , 0 7 0 4}$ & $\mathbf{6 , 5 6 9 3}$ & $\mathbf{0 , 8 5 5 1 ; 5 0 , 4 6 6 7}$ & $\mathbf{0 , 0 7 0 4}$ \\
\hline
\end{tabular}

mesmo com uma taxa de recidiva alta $(72,72 \%)$ entre os pacientes que sofreram uma primeira ressecção R0, as taxas de ressecabilidade $(81,25 \%)$ e de radicalidade entre os ressecados $(61,53 \%)$ na primeira re-ressecção foram pouco diferentes das taxas gerais da ressecção inicial (76,27 e 48,88\%, respectivamente). Esses índices caíram na segunda e terceira re-ressecções e não houve óbito nas re-ressecções. Tanto a ressecção radical $\mathrm{R} 0$ e a re-ressecção nos casos de recidiva ou persistência de doença foram positivamente significativas quanto ao prognóstico (Tabela 2), dados também encontrados por Lewis et al ${ }^{10}$.

$\mathrm{Na}$ análise do tratamento adjuvante não identificamos uma relação de indicação quanto a tipo histológico, grau de diferenciação celular, diâmetro do tumor ou radicalidade cirúrgica, uma vez que são estes os critérios tradicionais para indicação de quimioterapia adjuvante. Já o tratamento neoadjuvante foi realizado em $40 \%$ dos rabdomiossarcomas e em $100 \%$ dos tumores neuroectodérmicos primitivos (PNET).

Não houve significância estatística quanto ao prognóstico nos pacientes que realizaram tratamento adjuvante, mas sim uma tendência para um pior prognóstico nestes pacientes, em conformidade com a literatura ${ }^{10,11}$.

Os tumores apresentavam grande diâmetro, com média de 20,4 cm (quatro a $52 \mathrm{~cm}$ ), o que representa uma área de superfície tumoral de cerca de $1307,4 \mathrm{~cm}^{2}$ e dificultou muito a avaliação histopatológica de todos os limites, especialmente se levarmos em conta que uma lâmina de microscópio tem cerca de $1 \mathrm{~cm}^{2}$. Dificultou também a avaliação da margem adequada na proximidade de grandes vasos e a realização da cirurgia compartimental, a exemplo dos sarcomas de extremidade. Há uma tendência histórica no serviço de cirurgia abdomino-pélvica do INCA em realizar a cirurgia compartimental para os sarcomas retroperitoniais, evitando a lise de aderências entre o tumor e os órgãos adjacentes, no intuito de evitar uma eventual fratura, violação e conseqüente disseminação tumoral. Devido a este raciocínio e as grandes massas encontradas, em $64,44 \%$ dos pacientes ressecados, foram realizadas ressecções associadas de outros órgãos, estando somente $37,14 \%$ destes realmente invadidos na avaliação histopatológica. Essas ressecções associadas aumentaram a morbidade e não tiveram significância quanto ao prognóstico (Tabela2).

O diâmetro médio dos tumores analisados foi muito superior ao ponto de corte de $10 \mathrm{~cm}$ determinado na literatu$\mathrm{ra}^{10}$, só havendo em nossa série quatro pacientes com tumores menores que $10 \mathrm{~cm}$. Optamos por determinar como ponto de corte para análise estatística aquele que representasse, no mínimo, 30\% da amostra, sendo então determinado o diâmetro de $12 \mathrm{~cm}$, o que se mostrou significativo para um melhor prognóstico, quando igual ou inferior a esse diâmetro (Tabela 2).

Nos pacientes submetidos à hemotransfusão, o tempo operatório foi, uma hora e 30 minutos $(37,5 \%)$ maior que a média geral, possivelmente pela maior dificuldade cirúrgica, aderência e/ou proximidade de grandes vasos. Cabe supor que este pior prognóstico possa ser devido a uma maior dificuldade cirúrgica, com maior tempo operatório ou devido ao efeito imunossupressor inerente à hemotransfusão. Nossa opinião é que provavelmente haja uma associação destes fatores.

A morbidade foi inferior à média geral do serviço ${ }^{8,9}$, com $20,33 \%$ de complicações pós-operatórias, que geraram quatro $(8,9 \%)$ reoperações durante a mesma internação da operação principal. A mortalidade nos primeiros 30 dias de pós-operatório ocorreu em dois casos $(3,38 \%)$, um por sangramento e outro por embolia pulmonar, ambos em ressecções paliativas.

Assim como na literatura ${ }^{2,10,12,13}$, os tumores mais freqüentes foram os leiomiossarcomas e os lipossarcomas $(52,54 \%)$ embora o tipo histológico não tenha sido significativo para o prognóstico em conformidade com o descrito por outros autores ${ }^{10,11,14}$.

Os tumores pouco diferenciados (G3) $(38,98 \%)$ foram os mais comuns e, quando comparados em associação com os G4, com a associação dos G1 e G2, determinaram pior prognóstico na análise estatística (Tabela 2), fato também descrito por vários autores $3,11,14,15$.

A análise multivariada das variáveis com significância estatística na análise univariada, ou seja, diâmetro do tumor, grau de diferenciação celular, hemotransfusão, radicalidade da ressecção e re-ressecção,revelou que somente a reressecção se mostrou com tendência para variável independente, $\operatorname{com} p=0,0704$ (Tabela 3), demonstrando não haver variáveis independentes neste estudo.

A avaliação do padrão de recidiva dos pacientes que sofreram ressecções radicais R0 (22 pacientes), evidenciou um padrão amplo de recidiva, que ocorreu por via hematogênica (sete casos), celômica (seis casos), local (quatro casos) e linfática (dois casos), sendo três pacientes com recidiva em dois padrões distintos e concomitantes, não seguindo completamente o padrão celômico e local descrito na literatura ${ }^{3,16}$.

Tanto a sobrevida global quanto a sobrevida livre de doença foram inferiores às da literatura, provavelmente pelo 
fato de $93,22 \%$ dos nossos pacientes apresentarem diâmetro $>$ que $10 \mathrm{~cm}$ (33), 35,59\% terem sofrido hemotransfusão, termos incluído os óbitos pós-operatórios no cálculo da sobrevida e $72,88 \%$ terem grau de diferenciação tumoral G3, G4 ou GX.

As baixas taxas de controle e o caráter recidivante dos sarcomas deixam claro que o tratamento cirúrgico exclusivo não é o suficiente para garantir uma sobrevida a longo prazo, mesmo após cirurgias radicais em nossa série de pacientes.
Nessa série não encontramos vantagem em se ressecar órgãos adjacentes ou na cirurgia compartimental, devendo ser esta realizada, em grandes massas, somente quando houver forte suspeita de invasão tumoral direta, com comprometimento da radicalidade da cirurgia.

Essas análises sugerem que, no momento, somente a associação do diagnóstico precoce, da cirurgia radical R0, da ausência de hemotransfusão intra-operatória e da re-ressecção nos casos de recidiva ou persistência de doença darão a possibilidade de uma sobrevida a longo prazo.

\begin{abstract}
Background: To evaluate the morbimortality, survival and prognostics factors of retroperitonial sarcomas. Methods: Retrospective analysis of 59 patients operated on at the Abdomino-Pelvic Department of Surgery of the National Cancer Institute of Brazil between June 1992 and July 2003. Results: The most common complaints were abdominal pain and abdominal mass. Resectability rate was $74.57 \%$, and radicality $48.88 \%$. There were two postoperative deaths (3.38\%) and 12 complications $(20.33 \%)$. Liposarcomas and leiomyosarcomas were the most common tumor types. The most frequent tumor grade was G3 (38.98\%) and tumor median diameter was $20.4 \mathrm{~cm}$. The overall two and five-year survival rates were $49 \%$ and $20 \%$ respectively and the median disease free survival rate was 23 months. In the univariate analysis, tumor diameter $(>$ or $<=$ $12 \mathrm{~cm})$, tumor differentiation grade $([G 1+G 2] X[G 3+G 4])$, radical $(R 0)$ or palliative resection $(R 1+R 2)$, operative blood transfusion, and re-resection, even if palliative, in cases of recurrence or persistence of disease $(n=52)$, were significant ( $p=0.0267,0.048,0.0001,0.022$ and 0.0003, respectively). Multivariate analysis did not show any independent factor. Conclusion: In the present moment, only early diagnosis, radical surgery, absence of intra-operative blood transfusion and reresection in cases of recurrence or persistence of disease, will provide greater chances of a long term survival.
\end{abstract}

Key Words: Sarcoma; Retroperitoneal neoplasms/surgery; Indicators of morbidity and mortality; Survival rate; Prognosis.

\section{REFERÊNCIAS}

1. Mettlin C, Priore R, Rao U, et al. Results of the national softtissue sarcoma registry. J Surg Oncol.1982;19(4):224-7.

2. Arlen M, Marcove RC. Retroperitoneal sarcomas. In: Arlen M, Marcove RC, editors. Surgical management of soft tissue sarcomas. Philadelphia: WB Saunders; 1987. p. 220.

3. Zornig C, Weh HJ, Krull A, et al. Retroperitoneal sarcoma in a series of 51 adults. Eur J Surg Oncol.1992;18(5):475-80.

4. Herman K, Kusy T. Retroperitoneal sarcoma-the continued challenge for surgery and oncology. Surg Oncol.1998;7(1-2):7781.

5. Lewis JJ, Benedetti F. Adjuvant theraphy for soft tissue sarcomas. Surg Oncol Clin North Am.1997;6(4):847-62.

6. Braasch JW, Mon AB. Primary retroperitoneal tumors. Surg Clin North Am.1967;47(3):663-78.

7. Dalton RR, Donohue JH, Mucha P, et al. Management of retroperitoneal sarcomas. Surgery.1989;106(4):725-33

8. Corrêa JHS, Farina R, Santos CER, et al. Análise de morbi-mortalidade em um serviço de cirurgia oncológica. In: Congresso Brasileiro de Cirurgia, 24, 2001, São Paulo. Anais... São Paulo, Colégio Brasileiro de Cirurgiões, 2001, p. 320.

9. Corrêa JHS, Farina R, Santos CER, et al. Experiência do programa de gestão pela qualidade total (PGQT) em um serviço de cirurgia oncológica. In: Congresso Brasileiro de Cirurgia, 24, 2001, São Paulo. Anais... São Paulo, Colégio Brasileiro de Cirurgiões, 2001, p. 322.
10. Lewis JJ, Leung D, Woodruff JM, et al. Retroperitoneal softtissue sarcoma: analysis of 500 patients treated and followed at a single institution. Ann Surg. 1998;228(3):355-65.

11. Singer S, Corson JM, Demetri GD, et al. Prognostic factors predictive of survival for truncal and retroperitoneal soft-tissue sarcoma. Ann Surg.1995; 221(2):185-95.

12. Stoeckle E, Coindre JM, Bonvalot, et al. Prognostic factors in retroperitoneal sarcoma: a multivariate analysis of a series of 165 patients of the French Cancer Center Federation Sarcoma Group. Cancer.2001;92(2):359-68.

13. Storm FK, Eilber FR, Mirra J, et al. Retroperitoneal sarcomas: a reappraisal of treatment. J Surg Oncol.1981;17(1):1-7.

14. Herman K, Gruchala A, Niezabitowiski A, et al. Prognostic factors in retroperitoneal sarcomas: ploidy of DNA as a predictor of clinical outcome. J Surg Oncol. 1999;71(1):32-5.

15. Karakousis CP, Velez AF, Gerstenbluth R, et al. Resectability and survival in retroperitoneal sarcomas. Ann Surg Oncol. 1996;3(2):150-8.

16. Miraldi F, Adler LP, Faulhaber P. PET imaging in soft tissue sarcomas. Cancer Treat Res.1997;91:51-64.

Endereço para correspondência:

Carlos Eduardo Rodrigues Santos

Rua Macedo Sobrinho, 38/303

Humaitá - Rio de Janeiro - Rj

CEP: 22271-080 